\title{
Padé approximants for the ground-state energy of closed-shell quantum dots
}

\author{
Augusto Gonzalezt \\ Instituto de Cibernetica, Matematica y Fisica Calle E 309, Vedado, Habana 4, Cuba \\ Universidad Nacional, Sede Medellin AA 3840, Medellin, Colombia \\ Bart Partoens $\mathrm{Q}$ and François M. Peeters \\ Departement Natuurkunde, Universiteit Antwerpen (UIA) Universiteitsplein 1, B - 2610 Antwerpen, Belgium
}

(July 7, 2018)

\begin{abstract}
Analytic approximations to the ground-state energy of closed-shell quantum dots (number of electrons from 2 to 210) are presented in the form of two-point Padé approximants. These Padé approximants are constructed from the small- and large-density limits of the energy. We estimated that the maximum error, reached for intermediate densities, is less than $\leq 3 \%$. Within the present approximation the ground-state is found to be unpolarized.

PACS numbers: 73.20.Dx, 73.20.-r, 71.45.Gm
\end{abstract}

Quantum dots are artificial atoms which have been a subject of intense theoretical and experimental research in recent years. 1 They offer the very interesting possibility of varying the parameters characterising the dot in a broad range, and also of fixing at will the number of electrons, $N$, confined in the dot. External magnetic and electric fields can be added to generate new static and dynamical effects.

For very strong confinement (high electron densities) the one-particle Hamiltonian dominates over the coulomb repulsion, which can thus be treated as a perturbation. On the other hand, when the confinement is soft and the electron density becomes small, correlation effects start to play an important role, leading at very small densities to crystallisation, 2 as envisaged by Wigner. 3 Signals of the crystal phase can be seen even in the level structure of the $N=2-4$ systems in one dimension, 4 or in the "geometry" of the wave functions (the spatial distribution of the electron propability) of the three-electron system in two dimensions 5

In the present paper, we exploit the fact that the ground-state energy can be computed analytically in both the large- and small-density limits, from which we construct an estimate for the energy for arbitrary electron density by means of a two-point Padé approximant. The idea was applied lons ago to the three-dimensional (infinite) electron system 6 We have used it recently to compute the loy-lying energy levels of $N \leq 5$ electrons in a quantum dot, and is extended here to dots containing as many as 210 electrons.

One may ask why an interpolant should be a good approximation to the ground-state energy. The reason is that the energy is a very smooth function of the density. For small systems, it was noticed in Ref. 4 that the low- density picture is valid up to surprisingly high densities, and in Ref. 7 it was stressed that the regions of convergence of both limiting expansions for the energy overlap. Below, we will argue that these regions also overlap for larger $N$-values.

We study quantum dots consisting of $N$ electrons moving in two dimensions under the action of a parabolic potential. The Hamiltonian describing the system may be written in dimensionless form as

$$
h=\frac{H}{\hbar \omega_{0}}=\frac{1}{2} \sum_{i=1}^{N}\left(\vec{p}^{2}+\vec{r}^{2}\right)+\beta^{3} \sum_{i<j} \frac{1}{\left|\vec{r}_{i}-\vec{r}_{j}\right|}
$$

where $\omega_{0}$ is the dot frequency, $\beta^{3}=\sqrt{\left(\frac{\mu e^{4}}{\kappa^{2} \hbar^{2}}\right) /\left(\hbar \omega_{0}\right)}$ is a measure for the strength of the effective electron-electron interaction, $\mu$ is the electron effective mass, and $\kappa$ the dielectric constant of the material the electrons are moving in. We use the oscillator's natural units, i.e. $\sqrt{\hbar /\left(\mu \omega_{0}\right)}$ for length, $\hbar \omega_{0}$ for energy, etc. Notice that $\beta$ is the only parameter entering the hamiltonian $h$. By varying $\beta$ we modify the "density" of the system.

Following references, 8 we will construct two-point Padé approximants from the series expansions of the energy, $\epsilon$, in the $\beta \rightarrow 0$ and $\beta \rightarrow \infty$ limits

$$
\begin{aligned}
\left.\epsilon\right|_{\beta \rightarrow 0} & =b_{0}+b_{3} \beta^{3}+b_{6} \beta^{6}+\ldots, \\
\left.\epsilon\right|_{\beta \rightarrow \infty} & =\beta^{2}\left\{a_{0}+a_{2} / \beta^{2}+a_{4} / \beta^{4}+\ldots\right\},
\end{aligned}
$$

in which $b_{0}$ is the energy of $N$ free electrons in the quadratic potential, $b_{3}=<\Psi_{0}\left|\sum_{i<j}\right| \vec{r}_{i}-\left.\vec{r}_{j}\right|^{-1} \mid \Psi_{0}>$, $a_{0}$ is the classical (harmonic plus coulomb) energy of the $N$-electron system, $a_{2}=\sum_{i} \omega_{i} / 2$ is the zero-point energy of the classical cluster, i.e. the $\omega_{i}$ are the small-oscillation frequencies around the equilibrium configuration. $\beta \rightarrow 0$ will be called the oscillator limit, and $\beta \rightarrow \infty$ the Wigner limit indicating that a finite- $N$ analog of the Wigner crystal (a Wigner "cluster") is formed in this limit.

We computed the coefficients $b_{0}, b_{3}, a_{0}$ and $a_{2}$ for dots with up to 210 electrons. Higher coefficients could, in principle, be obtained although the calculations become rather involved. From these coefficients we may construct the following approximants interpolating between the expansions for $\beta \rightarrow 0$ and $\beta \rightarrow \infty$

$P_{3,2}(\beta)=b_{0}+a_{0} \beta^{2}\left\{1-\frac{1}{1+\left(b_{3} / a_{0}\right) \beta+\left(a_{0} /\left(b_{0}-a_{2}\right)\right) \beta^{2}}\right\}$, 


$$
\begin{aligned}
P_{4,3}(\beta) & =b_{0}+\frac{b_{3} \beta^{3}}{1+q_{1} \beta+q_{2} \beta^{2}+q_{3} \beta^{3}} \\
& +a_{0} \beta^{2}\left\{1-\frac{1+q_{1} \beta}{1+q_{1} \beta+q_{2} \beta^{2}+q_{3} \beta^{3}}\right\},
\end{aligned}
$$

where $q_{2}=a_{0} /\left(b_{0}-a_{2}\right), q_{1}=a_{0} q_{2} / b_{3}$, and $q_{3}=\left(a_{0} q_{1}-\right.$ $\left.b_{3}\right) /\left(b_{0}-a_{2}\right) . P_{3,2}$ and $P_{4,3}$ are the first two terms of the second-diagonal Padé sequence $\left\{P_{s+1, s}(\beta)\right\}$. The labels $s, t$ in $P_{s, t}$ mean that we are taking $s+1$ coefficients from the $\beta \rightarrow 0$ series and $t+1$ coefficients from the $\beta \rightarrow \infty$ series to construct the approximant. We will use (4) and (5) to estimate the ground-state energy of the $N$-electron dot.

In what follows, we restrict the analysis to closed-shell quantum dots, i.e. systems in which the first $N_{\text {shell }}$ oscillator shells are filled for $\beta=0$ which will simplify our calculation significantly. The number of electrons is thus restricted to the following values: $N=N_{\text {shell }}\left(N_{\text {shell }}+1\right)$. The energy in the $\beta=0$ limit is $b_{0}=N\left(2 N_{\text {shell }}+1\right) / 3$. For these systems, there is only one level starting from $\epsilon=b_{0}$ at $\beta=0$. Its angular momentum and spin are, respectively, $L=0$ and $S=0$. This will be the groundstate for small $\beta$. On the other hand, for very large values of $\beta$ the leading contributions to the energy (i.e. the coefficients $a_{0}$ and $a_{2}$ ) are independent of the spin state of the system. Thus, we may undoubtedly construct the small- $\beta$ and large- $\beta$ expansions for the lowest rotational invariant $(L=0)$, unpolarised $(S=0)$ state. In the rest of the paper, this will be called the "ground" state, al-

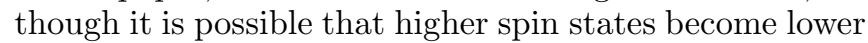
in energy for intermediate densities. 10

Let us compute the coefficient $b_{3}$. The wave function for $\beta=0$ is given by the Slater determinant

$$
\Psi_{0}=\left|\begin{array}{ccc}
\phi_{1}(1) \chi_{+}(1) & \ldots & \phi_{1}(N) \chi_{+}(N) \\
\phi_{1}(1) \chi_{-}(1) & \ldots & \phi_{1}(N) \chi_{-}(N) \\
\ldots & & \\
\phi_{N / 2}(1) \chi_{+}(1) & \ldots & \phi_{N / 2}(N) \chi_{+}(N) \\
\phi_{N / 2}(1) \chi_{-}(1) & \ldots & \phi_{N / 2}(N) \chi_{-}(N)
\end{array}\right|,
$$

where we numbered sequentially the harmonic wave functions (orbitals) $\phi_{1}, \ldots, \phi_{N / 2}$; and $\chi_{+}, \chi_{-}$are the spin-up and spin-down functions. The explicit form of the oneelectron orbitals is

$$
\phi_{k, l}=C_{k,|l|} r^{|l|} L_{k}^{|l|}\left(r^{2}\right) e^{-r^{2} / 2} e^{i l \theta},
$$

where $C_{k,|l|}=\sqrt{k ! /[\pi(k+|l|) !]}$, and for the composed index $n=(k, l)$ we defined the following sequential order: $\{(0,0)\},\{(0,1),(0,-1)\},\{(1,0),(0,2),(0,-2)\}, \ldots$, etc, i.e. increasing $|l|$ inside a shell with positive-l orbitals are taken first. The energy corresponding to $\phi_{k, l}$ is $\lambda_{k, l}=$ $1+2 k+|l|$. In terms of these energies we have $b_{0}=$ $\sum_{n} \lambda_{k, l}$. The coefficient $b_{3}$ can be written as

$b_{3}=\sum_{n_{1}} I\left(n_{1}, n_{1}\right)+4 \sum_{n_{1}<n_{2}} I\left(n_{1}, n_{2}\right)-2 \sum_{n_{1}<n_{2}} J\left(n_{1}, n_{2}\right)$, where

$$
\begin{aligned}
I\left(n_{(5)}, n_{2}\right) & =\int \frac{\mathrm{d}^{2} r_{1} \mathrm{~d}^{2} r_{2}}{\left|\vec{r}_{1}-\vec{r}_{2}\right|}\left|\phi_{n_{1}}\left(\vec{r}_{1}\right)\right|^{2}\left|\phi_{n_{2}}\left(\vec{r}_{2}\right)\right|^{2}, \\
J\left(n_{1}, n_{2}\right) & =\int \frac{\mathrm{d}^{2} r_{1} \mathrm{~d}^{2} r_{2}}{\left|\vec{r}_{1}-\vec{r}_{2}\right|} \phi_{n_{1}}^{*}\left(\vec{r}_{1}\right) \phi_{n_{2}}^{*}\left(\vec{r}_{2}\right) \phi_{n_{2}}\left(\vec{r}_{1}\right) \phi_{n_{1}}\left(\vec{r}_{2}\right),
\end{aligned}
$$

Both $I\left(n_{1}, n_{2}\right)$ and $J\left(n_{1}, n_{2}\right)$ can be reduced to sums of products of Gamma functions.

The numerical results for the coefficients $b_{3}$ are presented in Table 1 . Notice that a simple Thomas-Fermi estimate for the present problem 11 shows the scaling property: $\epsilon_{T F}(N, \beta)=N^{3 / 2} \epsilon_{T F}\left(1, N^{1 / 12} \beta\right)$ and, consequently, the $b_{3}$ coefficient should exhibit a $N^{7 / 4}$ behaviour in the large- $N$ limit which is satisfied by the results shown in Table I.

The classical energies of the $N$-electron clusters, i.e. the coefficients $a_{0}$, were computed by means $\rho^{f}$ a Monte Carlo technique with Newton optimisation 12.33 The values are also shown in Table [1. For large $N$, the Thomas-Fermi approximation predicts the dependence $a_{0} \approx 1.062 N^{5 / 3}$. We may obtain another estimate for $a_{0}$ from the classical energy of an hexagonal lattice structure, yielding $a_{0} \approx 0.968 N^{5 / 3}$. In Table $\mathbb{1}$, it is shown that the coefficients $a_{0}$ of the finite systems follow the $N^{5 / 3}$ law when $N \geq 30$.

Once the configuration of minimum energy of the classical problem is found, the small-oscillation problem is solved in order to compute the frequencies 13 and, consequently, to find $a_{2}$. The results are shown in the last column of Table I. A very rough estimate of the zeropoint energy is obtained from the dispersion relation of the longitudinal mode of an hexagonal lattice in the $(1,0)$ direction, yielding $a_{2} \approx 0.78 N^{5 / 4}$. The results in Table make evident the $N^{5 / 4}$ law for $N \geq 30$.

An evident conclusion that can be extracted from Table 1 is that the properties of quantum dots with $N>100$ are dictated by the $N \rightarrow \infty$ asymptotics. With other words, the ground-state energy for such a dot should be accurately described by a $P_{4,3}$ approximant in which $b_{0} \approx$ $\frac{2}{3} N^{3 / 2}, b_{3} \approx 0.7 N^{7 / 4}, a_{0} \approx 1.0 N^{5 / 3}$ and $a_{2} \approx 0.6 N^{5 / 4}$. In this sense, these dots are more "infinite" than "mesoscopic", and their properties will be very well reproduced by statistical theories.

Another very general property following from the results shown in Table 1 is the fact that the regions of convergence of the expansions (2) and (3) overlap. As an example, we show in Fig. 1] the $P_{4,3}$ approximant (solid curve) along with the small- $\beta$ and large- $\beta$ expansions for the energy (dashed curves) for the quantum dot with 42 electrons. The asymptotic curves almost intersect one another at $\beta \approx 0.8$. This smoothness of the dependence of $\epsilon$ on $\beta$ guarantees that an interpolant will be a very good approximation to the actual energy at any intermediate value of $\beta$. The same property was shown to hold for the ground-state and the excited levels of small dots with $N \leq 5$. For large dots, this seems to be a general property which follows 
from the scaling laws obeyed by the coefficients. Indeed, we can obtain a naive estimate of the radii of convergence of the series (2) and (3) by comparing consecutive terms. The small- $\beta$ expansion is expected to work when $\beta<\beta_{0}=\left(b_{0} / b_{3}\right)^{1 / 3} \approx N^{-1 / 12}$, while the large- $\beta$ expansion will be valid for $\beta>\beta_{\infty}=\left(a_{2} / a_{0}\right)^{1 / 2} \approx N^{-5 / 24}$. Because $\beta_{0} \geq \beta_{\infty}$, the regions of convergence will overlap.

We show in Fig. 2 the "convergence" of the Padé sequence, i.e. the relative difference between the $P_{4,3}$ and the $P_{3,2}$ approximants for the 42 -electron system. This figure suggests that the relative error of the $P_{4,3}$ approximant should not exceed $3 \%$ over the entire interval $0 \leq \beta<\infty$. The pattern is similar to the one encountered in small dots and therefore we expect this to be an estimate of the actual error.

Finally, we turn ourselves to the question of the absolute ground-state of the system. In the present approximation, the energy for large $\beta$ is independent of the polarisation state of the system. The leading approximation to the energy is again given by Eq. (2), where $a_{0}$ and $a_{2}$ are shown in Table 1 . For intermediate $\beta$ values, however, spatially antisymmetric states may be favoured as they minimise the Coulomb repulsion. We may easily compute the coefficients $b_{0}$ and $b_{3}$ at least for the lowest antisymmetric state at $\beta<<1$. This state is built up by placing one electron per orbital. If there are free orbitals in the last shell, the occupancy of the orbitals will lead to a maximal angular momentum (Hund's rule). For the coefficient $b_{3}$ we have now the expression

$$
b_{3}=\sum_{n_{1}<n_{2}}\left\{I\left(n_{1}, n_{2}\right)-J\left(n_{1}, n_{2}\right)\right\},
$$

where the sum runs over the occupied states.

In Table II, the angular momentum of such antisymmetric states and the coefficients $b_{0}$ and $b_{3}$ are given as function of $N$. Padé approximants are constructed in the same way as for unpolarised states.

We show in Fig. 3 a comparison between the estimated energies of the polarised and unpolarised states for the dot with 42 electrons. Both curves become very close for $\beta \geq 1$, but the unpolarised state is always the lowest state for any $\beta$. We found that this is the case for all N-values shown in Table II, which implies that in the present approximation the unpolarised state corresponds to the absolute ground-state for closed-shell quantum dots. This agrees with recent quantum mechanical density functional calculations.10

In conclusion, we have obtained Padé approximants to the ground-state energy of $N$ electrons $(2 \leq N \leq 210)$ which move in two dimensions and are confined by a parabolic potential. The approximants are asymptotically exact in both the high- and low-density limits. The maximum relative error for intermediate densities is estimated to be only a few percent of the actual energy. This is also a typical accuracy reached in density-fimctional calculations when the correlations are strong. 14.15
To increase the usefulness of the present results we were able to fit the coefficients in Table as follows

$$
\begin{aligned}
& a_{0} / N^{5 / 3}=1.062-0.875 / N^{1 / 2}-0.185 / N, \\
& a_{2} / N^{5 / 4}=0.573+0.475 / N^{1 / 2}-0.160 / N, \\
& b_{0} / N^{3 / 2}=2 / 3+0.083 / N, \\
& b_{3} / N^{7 / 4}=0.731-0.471 / N^{1 / 2}-0.046 / N .
\end{aligned}
$$

For $N \geq 6$ the fit to all the coefficients has a relative error which is smaller than $0.2 \%$.

In principle, the Padé-approximant method could equally well be applied to other dot configurations (i.e. non parabolic confinement, quasi-two-dimensional systems) or to-gther problems suchas for example, metallic clusters 1618 ions in traps 1921 etc. In these cases, more numerical work will be required to compute the coefficients $b_{0}, b_{3}, a_{0}$ and $a_{2}$.

Acknowledgments: A. G. acknowledges support from the International Centre for Theoretical Physics, Trieste, Italy, and from the Colombian Institute for Science and Technology (COLCIENCIAS). B.P. is an aspirant, and F.M.P. a research director with the Flemish Science Foundation (FWO Vlaanderen). Part of this work is supported by FWO and the 'Interuniversity Poles of Attraction Programme - Belgian State, Prime Minister's Office - Federal Office for Scientific, Technical and Cultural Affairs'.

* Associate Member of the International Centre for Theoretical Physics, Trieste, Italy.

Electronic mail: agonzale@perseus.unalmed.edu.co

- Electronic mail: bpartoen@uia.ua.ac.be

• Electronic mail: peeters@uia.ua.ac.be

${ }^{1}$ See, for example, J. H. Jefferson and W. Haüsler, condmat/9705012 and references therein.

${ }^{2}$ R. G. Clark, Physica Scripta T 39, 45 (1991).

${ }^{3}$ E. P. Wigner, Phys. Rev. 46, 1002 (1934).

${ }^{4}$ K. Jauregui, W. Haüsler, and B. Kramer, Europhys. Lett. 24, 581 (1993).

${ }^{5}$ P. A. Maksym, Phys. Rev. B 53, 10871 (1996).

${ }^{6}$ A. Ishihara and E. W. Montroll, Proc. Nat. Acad. Sci. 68, 3111 (1971).

${ }^{7}$ A. Gonzalez, J. Phys.: Cond. Matt. 9, 4643 (1997).

${ }^{8}$ A. Matulis and F. M. Peeters, J. Phys.: Cond. Matter 6, 7751 (1994).

${ }^{9}$ D. Ceperley, Phys. Rev. B 18, 3126 (1978).

${ }^{10}$ M. Koskinen, M. Manninen, and S. M. Reimann, Phys. Rev. Lett. 78 (1997).

${ }^{11}$ E. H. Lieb and J. P. Solovej, Phys. Rev. B 51, 10646 (1995).

${ }^{12}$ V. M. Bedanov and F. M. Peeters, Phys. Rev. B 49, 2667 (1994). 
${ }^{13}$ V. A. Schweigert and F. M. Peeters, Phys. Rev. B 51, 7700 (1995).

${ }^{14}$ M. Ferconi and G. Vignale, Phys. Rev. B 50, 14722 (1994).

${ }^{15}$ E. Lipparni, N. Barberan, M. Barranco, M. Pi, Ll. Serra, cond-mat/9704150.

16 W. A. de Heer, Rev. Mod. Phys. 65, 611 (1993).

${ }^{17}$ M. Brack, Rev. Mod. Phys. 65, 677 (1993).

${ }^{18}$ Comments Atom. Mol. Phys. 31, Nos. 3 - 6 (1995).

19 D.J. Wineland and W.M. Itano, Phys. Today 40(6), 34 (1987).

${ }^{20}$ S. Becker et al, Rev. Sci. Instr. 66, 4902 (1995).

${ }^{21}$ H. J. Kluge, Nucl. Instr. Methods Phys. Res. B 98, 500 (1995).

FIG. 1. Small- and large- $\beta$ expansions (dashed curves) and the $P_{4,3}$ approximant (solid curve) for the ground-state of the 42-electron system.

FIG. 2. Relative difference between the $P_{3,2}$ and the $P_{4,3}$ approximants for the 42-electron system.

FIG. 3. Energy estimates for the polarised (dashed curve) and unpolarised (solid curve) states for a quantum dot with 42 electrons.

TABLE I. The coefficients $b_{0}, b_{3}, a_{0}$ and $a_{2}$ for closed-shell quantum dots.

\begin{tabular}{|l|l|l|l|l|}
\hline \hline$N$ & $b_{0} / N^{3 / 2}$ & $b_{3} / N^{7 / 4}$ & $a_{0} / N^{5 / 3}$ & $a_{2} / N^{5 / 4}$ \\
\hline \hline 2 & 0.707107 & 0.372611 & 0.375 & 0.784567 \\
6 & 0.680414 & 0.531250 & 0.674147 & 0.738995 \\
12 & 0.673575 & 0.591525 & 0.793317 & 0.698756 \\
20 & 0.670820 & 0.623655 & 0.856344 & 0.671474 \\
30 & 0.669439 & 0.643709 & 0.895843 & 0.653428 \\
42 & 0.668648 & 0.657442 & 0.922532 & 0.639116 \\
56 & 0.668153 & 0.667452 & 0.941733 & 0.633269 \\
72 & 0.667823 & 0.675071 & 0.956422 & 0.626248 \\
90 & 0.667592 & 0.681068 & 0.967894 & 0.620456 \\
110 & 0.667424 & 0.685913 & 0.977103 & 0.616908 \\
132 & 0.667298 & 0.689909 & 0.984691 & 0.613269 \\
156 & 0.667201 & 0.693262 & 0.991046 & 0.610032 \\
182 & 0.667124 & 0.696118 & 0.996267 & 0.607227 \\
210 & 0.667063 & 0.698572 & 1.00107 & 0.605239 \\
\hline \hline
\end{tabular}

TABLE II. The angular momentum and the coefficients $b_{0}$ and $b_{3}$ for the lowest spatially antisymmetric (spin polarised) state.

\begin{tabular}{|l|l|l|l|}
\hline \hline$N$ & $J$ & $b_{0} / N^{3 / 2}$ & $b_{3} / N^{7 / 4}$ \\
\hline \hline 2 & 1 & 1.06066 & 0.186307 \\
6 & 0 & 0.952579 & 0.378007 \\
12 & 6 & 0.962250 & 0.428734 \\
20 & 5 & 0.950329 & 0.486891 \\
30 & 12 & 0.949386 & 0.508812 \\
42 & 18 & 0.947863 & 0.525187 \\
56 & 10 & 0.944959 & 0.538148 \\
72 & 36 & 0.946083 & 0.544753 \\
90 & 12 & 0.943998 & 0.555149 \\
\hline \hline
\end{tabular}


Figure 1

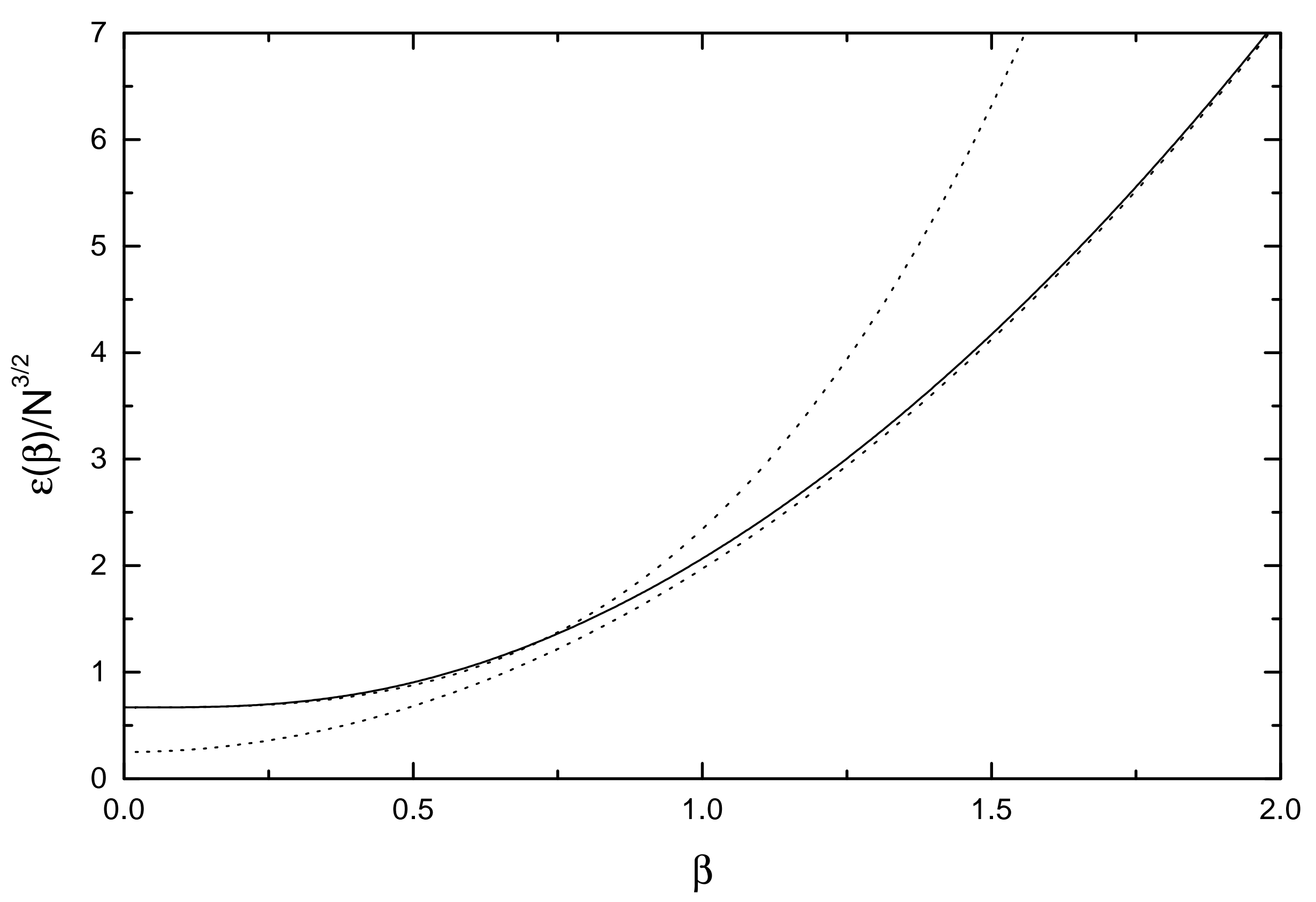


Figure 2

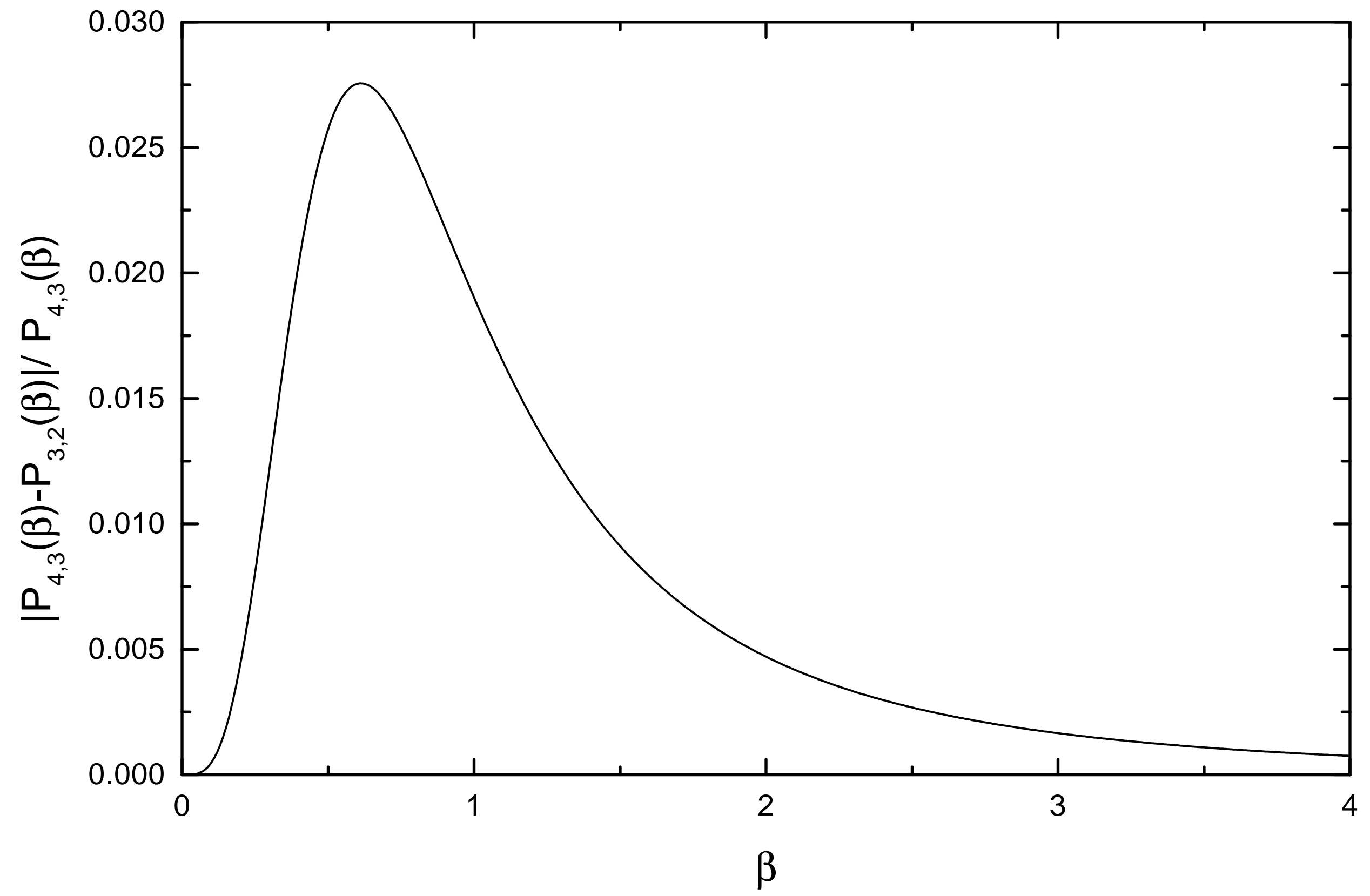


Figure 3

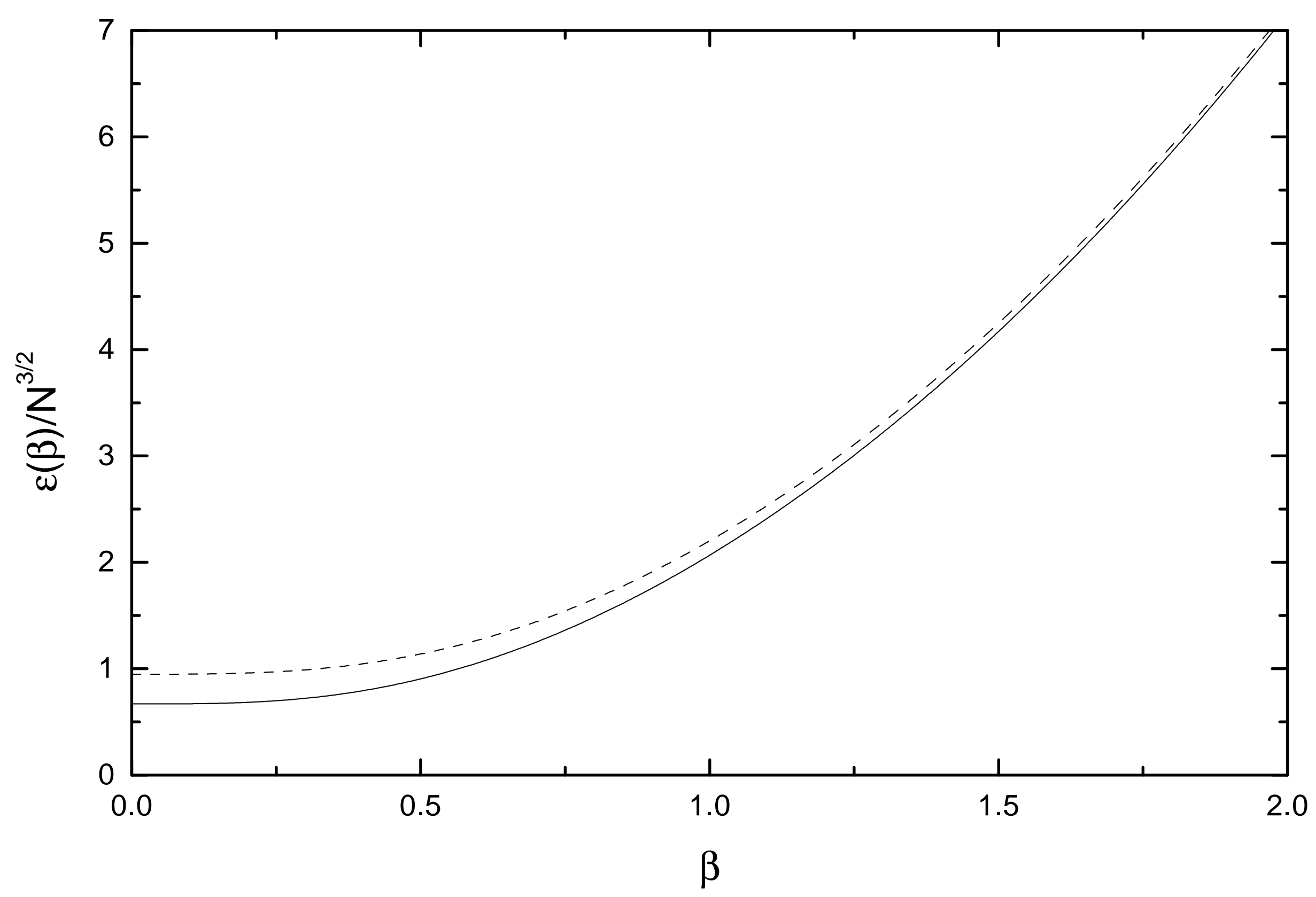

